NIST Technical Note 2079

\title{
Theoretical Basis of the Direct- Comparison System for Power Calibration Including Equivalent Source Mismatch
}

\author{
Jeffrey A. Jargon \\ Dazhen Gu \\ Christian J. Long \\ Aaron M. Hagerstrom \\ Angela C. Stelson \\ Ann F. Monke
}

This publication is available free of charge from:

https://doi.org/10.6028/NIST.TN.2079

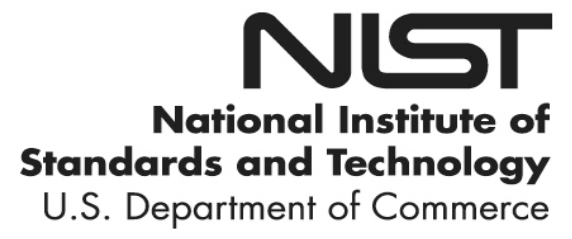


NIST Technical Note 2079

\title{
Theoretical Basis of the Direct- Comparison System for Power Calibration Including Equivalent Source Mismatch
}

\author{
Jeffrey A. Jargon \\ Dazhen $\mathrm{Gu}$ \\ Christian J. Long \\ Aaron M. Hagerstrom \\ Angela C. Stelson \\ Ann F. Monke \\ RF Technology Division \\ Communications Technology Laboratory
}

This publication is available free of charge from:

https://doi.org/10.6028/NIST.TN.2079

December 2019

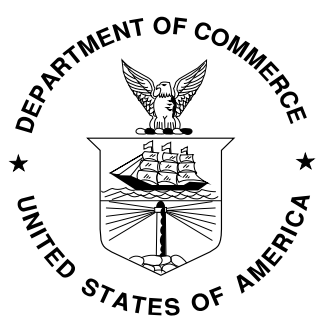

U.S. Department of Commerce Wilbur L. Ross, Jr., Secretary

National Institute of Standards and Technology Walter Copan, NIST Director and Undersecretary of Commerce for Standards and Technology 
Certain commercial entities, equipment, or materials may be identified in this document in order to describe an experimental procedure or concept adequately. Such identification is not intended to imply recommendation or endorsement by the National Institute of Standards and Technology, nor is it intended to imply that the entities, materials, or equipment are necessarily the best available for the purpose.

National Institute of Standards and Technology Technical Note 2079 Natl. Inst. Stand. Technol. Tech. Note 2079, 25 pages (December 2019) CODEN: NTNOEF

This publication is available free of charge from: https://doi.org/10.6028/NIST.TN.2079 


\begin{abstract}
In this report, we provide derivations for two equations that serve as the basis for the directcomparison system, a transfer apparatus for comparing microwave power sensors. The system consists of a synthesizer that provides a signal to the input of a power divider. A monitor power sensor is connected to one of the divider's output ports. During the first portion of the measurement, a transfer standard is connected to the other output port of the divider, and the indicated powers of the transfer standard and monitor are measured. Next, the transfer standard is replaced with an unknown power sensor to be calibrated, and the indicated powers of the unknown device and monitor are measured. Utilizing rules governing flow-diagrams for scattering-parameters and definitions of delivered power, we derive the effective efficiency of an unknown power sensor as a function of the transfer standard's effective efficiency in conjunction with power readings of both sensors and a monitor sensor, as well as reflection coefficients of the sensors and the equivalent source reflection coefficient of the power divider. Our derivation provides the definition of the equivalent source reflection coefficient. We also derive a correction term for the case when an adapter is connected to the transfer standard. Finally, we review the most widely-accepted approach for determining the equivalent source reflection coefficient of a three-port device, such as a power divider, and provide simplistic and comprehensive derivations that demonstrate its independence of the external impedances at two of its ports.
\end{abstract}

\title{
Key words
}

Calibration; derivation; direct-comparison system; equivalent source mismatch; power. 


\section{Table of Contents}

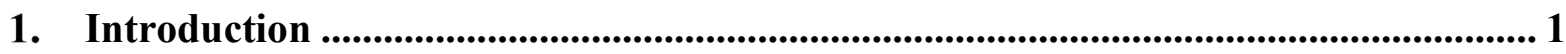

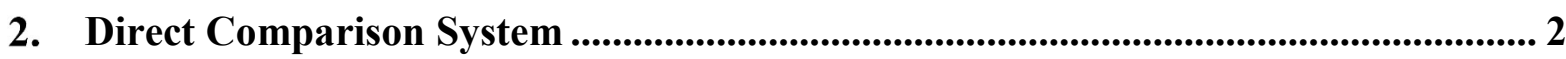

2.1. Adapter Correction ....................................................................................... 5

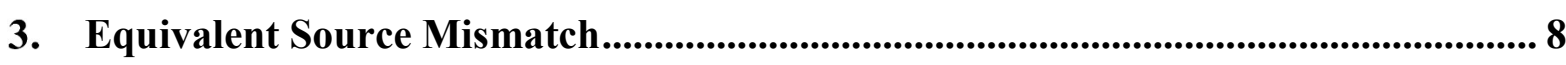

3.1. One-Port Error Model ............................................................................. 8

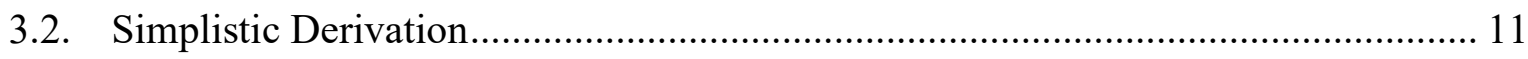

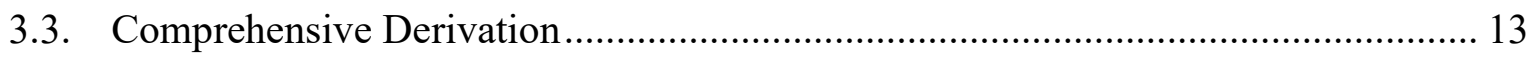

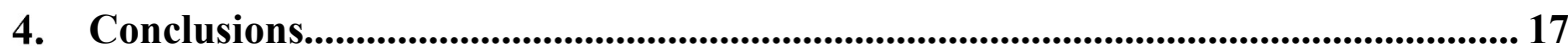

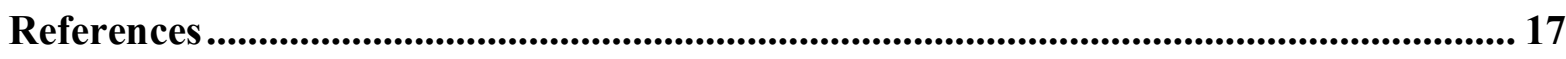




\section{List of Variables}

\begin{tabular}{|c|l|}
\hline Variable & \multicolumn{1}{c|}{ Definition } \\
\hline$a_{i,} b_{i}$ & Complex, frequency-domain waves at port i \\
\hline$a_{S}$ & Complex, frequency domain wave emanating from the source \\
\hline$e_{00}$ & Directivity error term of one-port VNA \\
\hline$e_{11}$ & Port match error term of one-port VNA \\
\hline$e_{10} e_{01}$ & Tracking error term of one-port VNA \\
\hline$P_{D}$ & Delivered power \\
\hline$P_{I}$ & Incident power \\
\hline$P_{M S}$ & $\begin{array}{l}\text { Measured dc-substituted power of monitor sensor with the } \\
\text { transfer standard connected }\end{array}$ \\
\hline$P_{M U}$ & $\begin{array}{l}\text { Measured dc-substituted power of monitor sensor with the } \\
\text { unknown sensor connected }\end{array}$ \\
\hline$S_{i j}$ & Scattering-parameter from port j to port i \\
\hline$\Gamma_{G}$ & Equivalent source mismatch of a three-port device \\
\hline$\Gamma_{M}$ & Reflection coefficient of the monitor sensor \\
\hline$\Gamma_{S}$ & Reflection coefficient of the transfer standard \\
\hline$\Gamma_{U}$ & Reflection coefficient of the unknown sensor \\
\hline$\Gamma^{\prime}$ & Measured reflection coefficient \\
\hline$\eta_{M}$ & Effective efficiency of the monitor sensor \\
\hline$\eta_{S}$ & Effective efficiency of the transfer standard \\
\hline$\eta^{\prime} S$ & $\begin{array}{l}\text { Effective efficiency of the transfer standard with an adapter } \\
\text { connected }\end{array}$ \\
\hline$\eta_{U}$ & Effective efficiency of the unknown sensor \\
\hline &
\end{tabular}




\section{Introduction}

The National Institute of Standards and Technology (NIST) offers calibration services for microwave power sensors and provides measurements of effective efficiency and calibration factor for thermistor, thermoelectric, and thin-film sensors. In the coaxial environment, measurements are implemented for GPC-7, Type-N, $3.5 \mathrm{~mm}, 2.92 \mathrm{~mm}$, and $2.4 \mathrm{~mm}$ connectors for frequencies between $100 \mathrm{kHz}$ and $50 \mathrm{GHz}$.

Although bolometric power sensors characterized by use of calorimeters serve as primary, traceable power standards [1], it is impractical to measure most sensors this way due to high costs, time constraints, and incompatible sensor designs. Most sensors are either not bolometric or do not have thermal properties required for measurements in a calorimeter. Thus, most coaxial power calibrations are performed using a direct-comparison approach, in which a test sensor is compared with a bolometric transfer standard that has previously been characterized by use of a calorimeter [2]. The advantage of this method is that it is based upon commerciallyavailable, wide-band resistive power dividers, or alternatively directional couplers in waveguide environments. The disadvantage is that mismatch corrections must be accounted for, so reflection coefficients of every device must be measured by use of a calibrated vector network analyzer (VNA).

A simplified schematic of a direct-comparison system is shown in Fig. 1. A synthesizer provides a continuous wave $(\mathrm{CW})$ signal to the input port (port 1) of a power divider. A monitor power sensor is connected to one of the divider's output ports (port 3 in this example). During the first portion of the measurement, a transfer standard is connected to the other output port of the divider (port 2 in this example), and the indicated powers of the transfer standard $P_{S}$ and monitor $P_{M}$ are measured as functions of frequency. Next, the transfer standard is replaced with an unknown power sensor to be calibrated, and the indicated powers of the unknown device $P_{U}$ and monitor $P_{M}$ are measured.

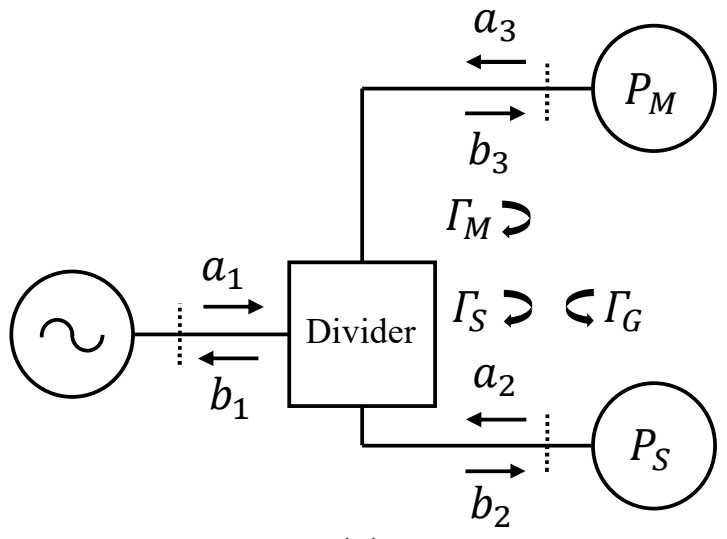

(a)

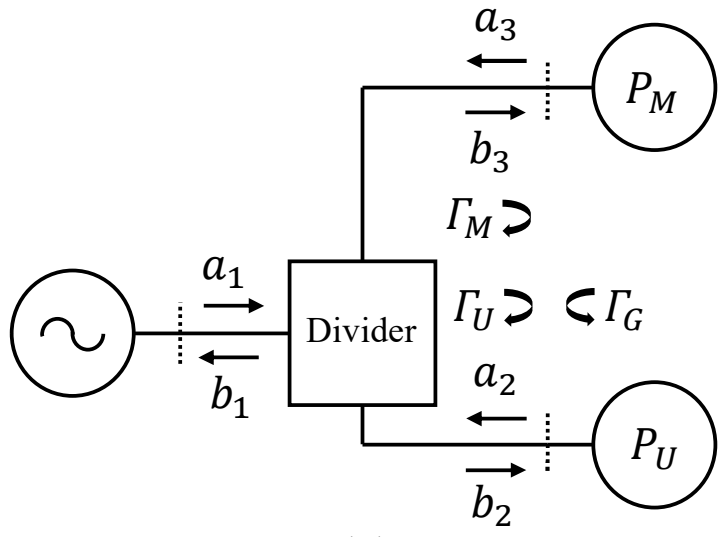

(b)

Fig. 1. Simplified schematic diagram of a direct-comparison system for power calibration: (a) with transfer standard connected to port 2, and (b) with unknown sensor connected to port 2. 
The effective efficiency of the unknown power sensor $\eta_{U}$ can be determined by use of the following equation [3]:

$$
\eta_{U}=\eta_{S} \frac{P_{M S}}{P_{M U}} \frac{P_{U}}{P_{S}} \frac{\left(1-\left|\Gamma_{S}\right|^{2}\right)}{\left(1-\left|\Gamma_{U}\right|^{2}\right)} \frac{\left|1-\Gamma_{G} \Gamma_{U}\right|^{2}}{\left|1-\Gamma_{G} \Gamma_{S}\right|^{2}},
$$

where the effective efficiency of the transfer standard $\eta_{S}$ is determined by use of a calorimeter. The reflection coefficients of the transfer standard $\Gamma_{S}$, unknown device $\Gamma_{U}$, and monitor sensor $\Gamma_{M}$ are determined by use of a one-port measurement with a calibrated vector network analyzer (VNA) [4], and $P_{M S}$ and $P_{M U}$ are the measured dc substituted powers of the monitor sensor with the transfer standard and unknown sensor connected, respectively. The equivalent source mismatch $\Gamma_{G}$ is defined in terms of the following calibrated $S$-parameters of the divider:

$$
\Gamma_{G} \stackrel{\text { def }}{=} S_{22}-\frac{S_{21} S_{32}}{S_{31}}
$$

and is measured using the technique presented in [5]. It turns out this term is a byproduct of the derivation of Eq. (1.1), as will be shown in the following section.

In this document, we provide derivations for Eq. (1.1) and Eq. (1.2), which serve as the basis for the direct-comparison system. In Sec. 2, we derive these two equations, as well as a correction term for the case when an adapter is connected to the transfer standard. In Sec. 3, we review the most widely-accepted approach for determining the equivalent source reflection coefficient of a three-port device, such as a power divider, and provide simplistic and comprehensive derivations that demonstrate its independence of the external impedances at two of its ports.

\section{Direct Comparison System}

In this section, we derive the two main equations governing the direct-comparison system for power calibration (Eq. (1.1) and Eq. (1.2)). We begin with the scattering-matrix representation of a three-port device [6], which represents the divider:

$$
\left[\begin{array}{l}
b_{1} \\
b_{2} \\
b_{3}
\end{array}\right]=\left[\begin{array}{lll}
S_{11} & S_{12} & S_{13} \\
S_{21} & S_{22} & S_{23} \\
S_{31} & S_{32} & S_{33}
\end{array}\right]\left[\begin{array}{l}
a_{1} \\
a_{2} \\
a_{3}
\end{array}\right]
$$

Here, $a_{i}$ and $b_{i}$ refer to complex, frequency-domain forward and backward waves normalized to a $50 \Omega$ reference impedance, and $S_{i j}$ represent the scattering-parameters ( $S$-parameters) relating the input at port $j$ to the output at port $i$. From Eq. (2.1), we can extract the equations for waves $b_{2}$ and $b_{3}$ :

$$
b_{2}=S_{21} a_{1}+S_{22} a_{2}+S_{23} a_{3}
$$

and 


$$
b_{3}=S_{31} a_{1}+S_{32} a_{2}+S_{33} a_{3} .
$$

When the transfer standard is connected to port 2 of the divider, as shown in Fig. 1, the waves at port 2 are related by

$$
a_{2}=\Gamma_{S} b_{2},
$$

where $\Gamma_{S}$ is the reflection coefficient of the transfer standard. Likewise, the waves at port 3 are related by

$$
a_{3}=\Gamma_{M} b_{3},
$$

where $\Gamma_{M}$ is the reflection coefficient of the monitor sensor. Substituting Eq. (2.4) and Eq. (2.5) into Eq. (2.2) and Eq. (2.3) gives

$$
b_{2}=S_{21} a_{1}+S_{22} \Gamma_{S} b_{2}+S_{23} \Gamma_{M} b_{3}
$$

and

$$
b_{3}=S_{31} a_{1}+S_{32} \Gamma_{S} b_{2}+S_{33} \Gamma_{M} b_{3} .
$$

Solving Eq. (2.6) and Eq. (2.7) in terms of $a_{1}$ results in

$$
a_{1}=\frac{1}{S_{21}}\left(b_{2}-S_{22} \Gamma_{S} b_{2}-S_{23} \Gamma_{M} b_{3}\right)
$$

and

$$
a_{1}=\frac{1}{S_{31}}\left(b_{3}-S_{32} \Gamma_{S} b_{2}-S_{33} \Gamma_{M} b_{3}\right)
$$

Equating Eq. (2.8) and Eq. (2.9) gives

$$
S_{31}\left(b_{2}-S_{22} \Gamma_{S} b_{2}-S_{23} \Gamma_{M} b_{3}\right)=S_{21}\left(b_{3}-S_{32} \Gamma_{S} b_{2}-S_{33} \Gamma_{M} b_{3}\right)
$$

or alternatively in terms of $b_{2}$ and $b_{3}$

$$
b_{2}\left(S_{31}-S_{22} S_{31} \Gamma_{S}+S_{21} S_{32} \Gamma_{S}\right)=b_{3}\left(S_{21}-S_{21} S_{33} \Gamma_{M}+S_{31} S_{23} \Gamma_{M}\right) .
$$

Solving for the ratio of $b_{2}$ over $b_{3}$ gives

$$
\frac{b_{2}}{b_{3}}=\frac{S_{21}-S_{21} S_{33} \Gamma_{M}+S_{31} S_{23} \Gamma_{M}}{S_{31}-S_{22} S_{31} \Gamma_{S}+S_{21} S_{32} \Gamma_{S}} .
$$

The power delivered to the transfer standard at port $2, P_{D 2}$, is given by [7] 


$$
P_{D 2}=\left|b_{2}\right|^{2}-\left|a_{2}\right|^{2}=\left|b_{2}\right|^{2}\left(1-\left|\Gamma_{S}\right|^{2}\right)
$$

and the power delivered to the monitor sensor at port $3, P_{D 3}$, is given by

$$
P_{D 3}=\left|b_{3}\right|^{2}-\left|a_{3}\right|^{2}=\left|b_{3}\right|^{2}\left(1-\left|\Gamma_{M}\right|^{2}\right) \text {. }
$$

Thus, the ratio of Eq. (2.13) and Eq. (2.14) is

$$
\frac{P_{D 2}}{P_{D 3}}=\frac{\left|b_{2}\right|^{2}}{\left|b_{3}\right|^{2}} \frac{\left(1-\left|\Gamma_{S}\right|^{2}\right)}{\left(1-\left|\Gamma_{M}\right|^{2}\right)} .
$$

Substituting Eq. (2.12) into Eq. (2.15) gives

$$
\frac{P_{D 2}}{P_{D 3}}=\frac{\left|S_{21}-S_{21} S_{33} \Gamma_{M}+S_{31} S_{23} \Gamma_{M}\right|^{2}}{\left|S_{31}-S_{22} S_{31} \Gamma_{S}+S_{21} S_{32} \Gamma_{S}\right|^{2}} \frac{\left(1-\left|\Gamma_{S}\right|^{2}\right)}{\left(1-\left|\Gamma_{M}\right|^{2}\right)} .
$$

The effective efficiency $\eta$ of a power sensor is defined as the ratio of the substituted power, determined by the electronics of the power meter, over the delivered power. Thus, the effective efficiency of the transfer standard $\eta_{S}$ is

$$
\eta_{S}=P_{S} / P_{D 2}
$$

where $P_{S}$ is the dc substituted power of the transfer standard [8]. Solving for the delivered power gives

$$
P_{D 2}=P_{S} / \eta_{S}
$$

Likewise, the effective efficiency of the monitor sensor $\eta_{M}$ is

$$
\eta_{M}=P_{M S} / P_{D 3}
$$

where $P_{M S}$ is the de substituted power of the monitor sensor when the transfer standard is connected to port 2. Solving for the delivered power gives

$$
P_{D 3}=P_{M S} / \eta_{M}
$$

Substituting Eq. (2.18) and Eq. (2.20) into Eq. (2.16) gives

$$
\frac{P_{S} / \eta_{S}}{P_{M S} / \eta_{M}}=\frac{\left|S_{21}-S_{21} S_{33} \Gamma_{M}+S_{31} S_{23} \Gamma_{M}\right|^{2}}{\left|S_{31}-S_{22} S_{31} \Gamma_{S}+S_{21} S_{32} \Gamma_{S}\right|^{2}} \frac{\left(1-\left|\Gamma_{S}\right|^{2}\right)}{\left(1-\left|\Gamma_{M}\right|^{2}\right)}
$$

A similar equation may be obtained when the unknown device is connected to port 2:

$$
\frac{P_{U} / \eta_{U}}{P_{M U} / \eta_{M}}=\frac{\left|S_{21}-S_{21} S_{33} \Gamma_{M}+S_{31} S_{23} \Gamma_{M}\right|^{2}}{\left|S_{31}-S_{22} S_{31} \Gamma_{U}+S_{21} S_{32} \Gamma_{U}\right|^{2}} \frac{\left(1-\left|\Gamma_{U}\right|^{2}\right)}{\left(1-\left|\Gamma_{M}\right|^{2}\right)},
$$


where $P_{U}$ is the de substituted power of the unknown device, $P_{M U}$ is the dc substituted power of the monitor sensor when the unknown device is connected to port $2, \eta_{U}$ is the effective efficiency of the unknown device, and $\Gamma_{U}$ is the reflection coefficient of the unknown device.

The ratio of Eq. (2.21) to Eq. (2.22) is

$$
\frac{\left(P_{S} / \eta_{S}\right)\left(P_{M U} / \eta_{M}\right)}{\left(P_{M S} / \eta_{M}\right)\left(P_{U} / \eta_{U}\right)}=\frac{\left|S_{31}-S_{22} S_{31} \Gamma_{U}+S_{21} S_{32} \Gamma_{U}\right|^{2}}{\left|S_{31}-S_{22} S_{31} \Gamma_{S}+S_{21} S_{32} \Gamma_{S}\right|^{2}} \frac{\left(1-\left|\Gamma_{S}\right|^{2}\right)}{\left(1-\left|\Gamma_{U}\right|^{2}\right)} .
$$

Solving for the effective efficiency of the unknown device gives

$$
\eta_{U}=\eta_{S} \frac{P_{M S}}{P_{M U}} \frac{P_{U}}{P_{S}} \frac{\left(1-\left|\Gamma_{S}\right|^{2}\right)}{\left(1-\left|\Gamma_{U}\right|^{2}\right)} \frac{\left|1-\left(S_{22}-S_{21} S_{32} / S_{31}\right) \Gamma_{U}\right|^{2}}{\left|1-\left(S_{22}-S_{21} S_{32} / S_{31}\right) \Gamma_{S}\right|^{2}},
$$

where $\Gamma_{G}$ may be defined as [9]

$$
\Gamma_{G} \stackrel{\text { def }}{=} S_{22}-\frac{S_{21} S_{32}}{S_{31}}
$$

This term is commonly referred to as the equivalent source mismatch [10]. Equation Eq. (2.24) can then be rewritten as

$$
\eta_{U}=\eta_{S} \frac{P_{M S}}{P_{M U}} \frac{P_{U}}{P_{S}} \frac{\left(1-\left|\Gamma_{S}\right|^{2}\right)}{\left(1-\left|\Gamma_{U}\right|^{2}\right)} \frac{\left|1-\Gamma_{G} \Gamma_{U}\right|^{2}}{\left|1-\Gamma_{G} \Gamma_{S}\right|^{2}}
$$

Equations Eq. (2.25) and Eq. (2.26) provide the basis of the direct-comparison system for power calibration.

\subsection{Adapter Correction}

Oftentimes, during the calibration of a power sensor, an adapter is required to make a connection between the transfer standard and the divider [11, 12], as illustrated in Fig. 2. This requires corrections be made to the reflection coefficient $\Gamma_{S}$ and effective efficiency $\eta_{S}$ to account for the $S$-parameters of the adapter. In this subsection, we derive equations for the corrected values, $\Gamma^{\prime} S$ and $\eta^{\prime}$ s. The waves at port 1 of the adapter are related by

$$
b_{1}=\Gamma_{S}^{\prime} a_{1}
$$

Next, solving for $b_{1}$ and $b_{2}$, illustrated in the flow diagram of Fig. 2, gives

$$
b_{1}=S_{11}^{A} a_{1}+S_{12}^{A} a_{2}
$$

and

$$
b_{2}=S_{21}^{A} a_{1}+S_{22}^{A} a_{2} .
$$


The waves at port 2 of the adapter are related by

$$
a_{2}=\Gamma_{S} b_{2}
$$

Substituting Eq. (2.30) into Eq. (2.29) gives

$$
a_{2} / \Gamma_{S}=S_{21}^{A} a_{1}+S_{22}^{A} a_{2}
$$

or

$$
a_{2}=\frac{S_{21}^{A} \Gamma_{S} a_{1}}{1-S_{22}^{A} \Gamma_{S}}
$$

Substituting Eq. (2.32) into Eq. (2.28) gives

$$
b_{1}=S_{11}^{A} a_{1}+\frac{S_{12}^{A} S_{21}^{A} \Gamma_{S} a_{1}}{1-S_{22}^{A} \Gamma_{S}} .
$$

Dividing both sides of Eq. (2.33) by $a_{1}$ and substituting the result into Eq. (2.27) gives the corrected value of $\Gamma^{\prime}$, the reflection coefficient of the adapter terminated with the transfer standard:

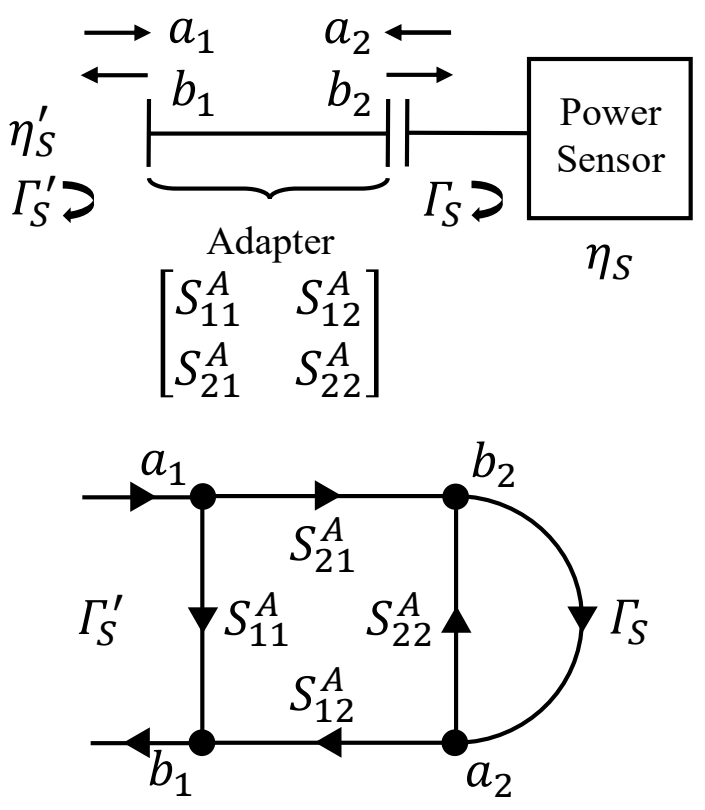

Fig. 2. The upper portion depicts an adapter connected to the transfer standard, and the lower portion depicts the flow diagram including reflection coefficients and $S$-parameters of the adapter. 


$$
\Gamma_{S}^{\prime}=S_{11}^{A}+\frac{S_{12}^{A} S_{21}^{A} \Gamma_{S}}{1-S_{22}^{A} \Gamma_{S}} .
$$

Next, we solve for the corrected value of $\eta^{\prime} s$, the effective efficiency of the adapter terminated with the transfer standard. We begin by substituting Eq. (2.30) into Eq. (2.29) and solving for $b_{2}$, which gives

$$
b_{2}=\frac{S_{21}^{A}}{1-S_{22}^{A} \Gamma_{S}} a_{1}
$$

The power incident on the adapter at port $1, P^{\prime}{ }_{1}$, is given by [7]

$$
P_{I}^{\prime}=\left|a_{1}\right|^{2}
$$

The power delivered to the adapter at port $1, P_{D}^{\prime}$, is given by

$$
P_{D}^{\prime}=P_{I}^{\prime}\left(1-\left|\Gamma_{S}^{\prime}\right|^{2}\right)=\left|a_{1}\right|^{2}\left(1-\left|\Gamma_{S}^{\prime}\right|^{2}\right) \text {. }
$$

The power incident on the transfer standard at port $2, P_{I}$, is given by

$$
P_{I}=\left|b_{2}\right|^{2}
$$

Substituting Eq. (2.35) into Eq. (2.38) gives

$$
P_{I}=\frac{\left|S_{21}^{A}\right|^{2}\left|a_{1}\right|^{2}}{\left|1-S_{22}^{A} \Gamma_{S}\right|^{2}} .
$$

The power delivered to the transfer standard at port $2, P_{D}$, is given by

$$
P_{D}=P_{I}\left(1-\left|\Gamma_{S}\right|^{2}\right)=\frac{\left|S_{21}^{A}\right|^{2}\left|a_{1}\right|^{2}}{\left|1-S_{22}^{A} \Gamma_{S}\right|^{2}}\left(1-\left|\Gamma_{S}\right|^{2}\right) .
$$

As mentioned in Sec. 2, the effective efficiency of a power sensor is defined as the ratio of the substituted power over the delivered power. Thus, the effective efficiency of the transfer standard at port 2 , denoted as $\eta_{S}$, is given by

$$
\eta_{S}=\frac{P_{D C}}{P_{D}}
$$

and the effective efficiency at port 1 , denoted as $\eta^{\prime} S$, is given by

$$
\eta_{S}^{\prime}=\frac{P_{D C}}{P_{D}^{\prime}}
$$

Solving Eq. (2.41) and Eq. (2.42) for $P_{D C}$ and equating them gives 


$$
\eta_{S}^{\prime} P_{D}^{\prime}=\eta_{S} P_{D}
$$

Solving Eq. (2.43) for $\eta^{\prime} s$ gives

$$
\eta_{S}^{\prime}=\eta_{S} \frac{P_{D}}{P_{D}^{\prime}}
$$

Substituting Eq. (2.37) and Eq. (2.40) into Eq. (2.44) allows us to solve for the corrected value of $\eta^{\prime} S$, the effective efficiency of the adapter terminated with the transfer standard as a function of $\eta_{S}, \Gamma_{S}, \Gamma_{S}^{\prime}$, and the $S$-parameters of the adapter:

$$
\eta_{S}^{\prime}=\eta_{S} \frac{1-\left|\Gamma_{S}\right|^{2}}{1-\left|\Gamma_{S}^{\prime}\right|^{2}} \frac{\left|S_{21}^{A}\right|^{2}}{\left|1-S_{22}^{A} \Gamma_{S}\right|^{2}} .
$$

Equation (2.45) is identical to that presented in [12].

An alternative formulation, which has been historically used internally at NIST, can be written as the effective efficiency of the transfer standard $\eta_{S}$ as a function of $\eta^{\prime} S, \Gamma_{S}$, and the $S$ parameters of the adapter. This may be accomplished by substituting Eq. (2.34) into Eq. (2.45) and solving for $\eta_{S}$, which gives

$$
\eta_{S}=\eta_{S}^{\prime} \frac{\left[\left|1-S_{22}^{A} \Gamma_{S}\right|^{2}-\left|\left(S_{12}^{A} S_{21}^{A}-S_{11}^{A} S_{22}^{A}\right) \Gamma_{S}+S_{11}^{A}\right|^{2}\right]}{\left|S_{21}^{A}\right|^{2}\left(1-\left|\Gamma_{S}\right|^{2}\right)} .
$$

In this section, we have derived the two equations, Eq. (2.25) and Eq. (2.26), that serve as the basis for the direct-comparison system, as well as a correction term, alternatively Eq. (2.45) or Eq. (2.46), for the case when an adapter is connected to the transfer standard.

\section{Equivalent Source Mismatch}

From Eq. (2.25), we can see that the equivalent source mismatch is exclusively a function of the $S$-parameters of the power divider and invariant of the signal generator and power sensors connected to it. Although various techniques have been developed to determine this quantity, the most widely-accepted approach is the method developed by Juroshek [5], which makes use of a one-port VNA calibration.

In Sec. 3.1, we review this method. Then, in the following sections, we provide simplistic and comprehensive derivations.

\subsection{One-Port Error Model}

The upper portion of Fig. 3 illustrates a simplified diagram of a one-port VNA, which consists of a signal generator, two directional couplers connected back-to-back, and an unknown oneport device. Since the measurement system is not perfect, the reflection coefficient measured by the VNA must be corrected with a calibration. Imperfections in the VNA are modeled by

taking the linear errors and combining them into a two-port error box between the couplers and 
the unknown one-port device. Since ratio measurements are taken, only three terms are required. The lower portion of Fig. 3 shows the flow graph of the two-port error box connected to the unknown reflection coefficient. The measured reflection coefficient, $\Gamma^{\prime}$, is mathematically related to the actual reflection coefficient, $\Gamma$, by three error terms: directivity $e_{00}$, port match $e_{11}$, and tracking $e_{10} e_{01}$. These three terms can be determined with measurements of three known calibrations artifacts such as an open, short, and load.

Solving the one-port flow graph in Fig. 3 results in a bilinear relationship between the measured reflection coefficient

$$
\Gamma^{\prime}=b_{0} / a_{0}
$$

and the actual reflection coefficient

$$
\Gamma=\frac{a_{1}}{b_{1}}=\frac{\left(b_{0} / a_{0}\right)-e_{00}}{\left(b_{0} / a_{0}\right) e_{11}-\Delta_{e}}=\frac{\Gamma^{\prime}-e_{00}}{\Gamma^{\prime} e_{11}-\Delta_{e}},
$$

where

$$
\Delta_{e}=e_{00} e_{11}-e_{10} e_{01}
$$

For the one-port VNA, $e_{11}$ is analogous to $\Gamma_{G}$ of the incident coupler (measuring $a_{0}$ ).
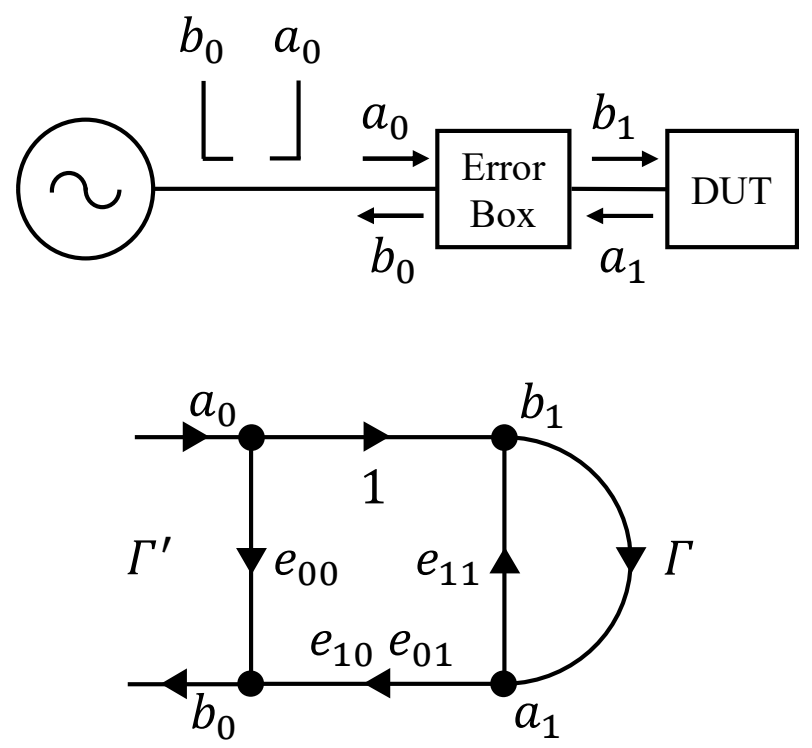

Fig. 3. The upper portion depicts a simplified diagram of a port VNA, and the lower portion depicts the flow diagram including the two-port error box that relates the measured reflection coefficient, $\Gamma^{\prime}$, to the actual reflection coefficient, $\Gamma$. 
Similarly, the scattering-parameters of a three-port device may be measured using a two-port VNA with the same technique [5]. Figure 4 illustrates such a setup, where the two-port VNA is connected to ports 1 and 3 of the divider, respectively.

In this configuration, $b^{\prime}{ }_{1}$ is a linear function of the signal reflected from port 1 of the divider and $b^{\prime} 3$ is a linear function of the signal reflected from port 3. Thus, as in Eq. (3.2), the actual reflection coefficient is

$$
\Gamma=\frac{b_{2}}{a_{2}}=\frac{\left(b_{1}^{\prime} / b_{3}^{\prime}\right)-e_{00}}{\left(b_{1}^{\prime} / b_{3}^{\prime}\right) e_{11}-\Delta_{e}}
$$

or

$$
\Gamma=\frac{b_{2}}{a_{2}}=\frac{\Gamma^{\prime}-e_{00}}{\Gamma^{\prime} \Gamma_{G}-\Delta_{e}},
$$

where

$$
\Gamma^{\prime}=b_{1}^{\prime} / b_{3}^{\prime} .
$$

Equation (3.5) can be solved by connecting three devices with known values of reflection coefficients to port 2 of the divider, where $\Gamma_{G}$ is the only term of interest, although $e_{00}$ and $\Delta_{e}$ are also unknowns that can be determined.

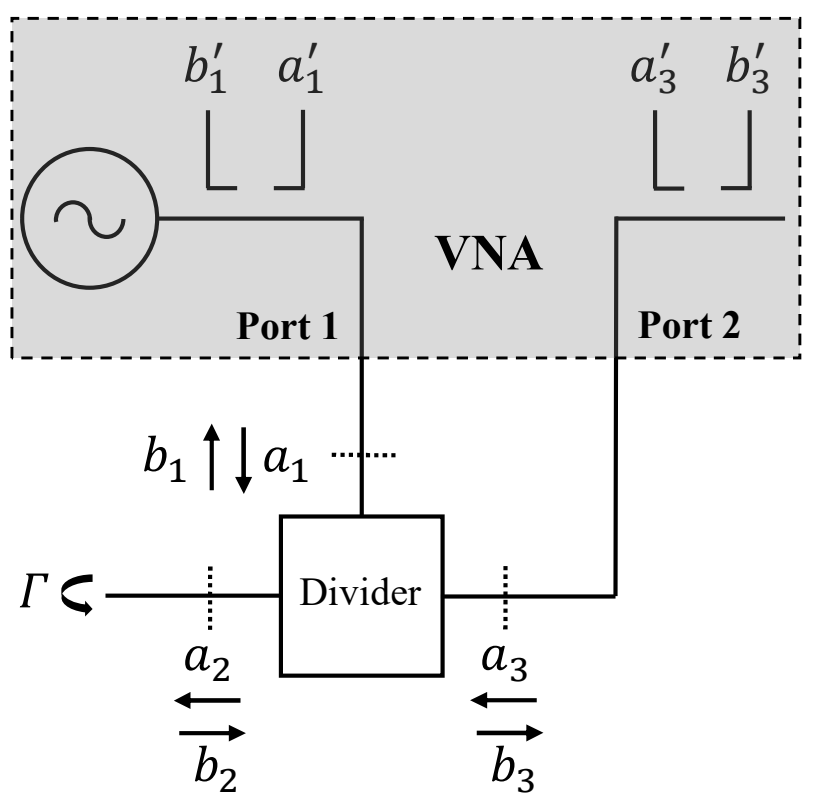

Fig. 4. Simplified diagram for measuring the equivalent source mismatch of a three-port device, such as a power divider, with a two-port VNA. 
Juroshek [5] summarizes the take-away from this method as:

"It is important to realize that devices with loss and/or reflection can be connected to either port 1 or port 3 of the coupler [in our case, divider] without changing $\Gamma_{G}$ assuming that the noise does not increase significantly due to the finite dynamic range of the VNA that measures $b_{1}^{\prime}$ and $b_{3}^{\prime}$. For example, an adapter with reasonable losses and reflections can be connected to port 1 without changing $\Gamma_{G}$ because the same change occurs in both $S_{13}$ and $S_{12}$ and, therefore, cancels out when the ratio is taken in Equation 3 [in our case (2.25)]. The same outcome is true for port 3 . However, the outcome will be different for port 2 and any such connection will affect $\Gamma_{G}$ directly. Thus, it is not important where $b_{1}^{\prime}$ and $b_{3}^{\prime}$ are measured as long as the dynamic range of the VNA is sufficient to measure them with the accuracy desired."

\subsection{Simplistic Derivation}

In this section, we derive Eq. (3.5) and Eq. (2.25) using a simplistic model consisting of a three-port device, where ports 1 and 3 are well-matched such that $\Gamma_{1}$ and $\Gamma_{3}$ are zero, as shown in Fig. 5.

We begin with the scattering-matrix representation of a three-port device, which represents the divider:

$$
\left[\begin{array}{l}
b_{1} \\
b_{2} \\
b_{3}
\end{array}\right]=\left[\begin{array}{lll}
S_{11} & S_{12} & S_{13} \\
S_{21} & S_{22} & S_{23} \\
S_{31} & S_{32} & S_{33}
\end{array}\right]\left[\begin{array}{l}
a_{1} \\
a_{2} \\
a_{3}
\end{array}\right] .
$$

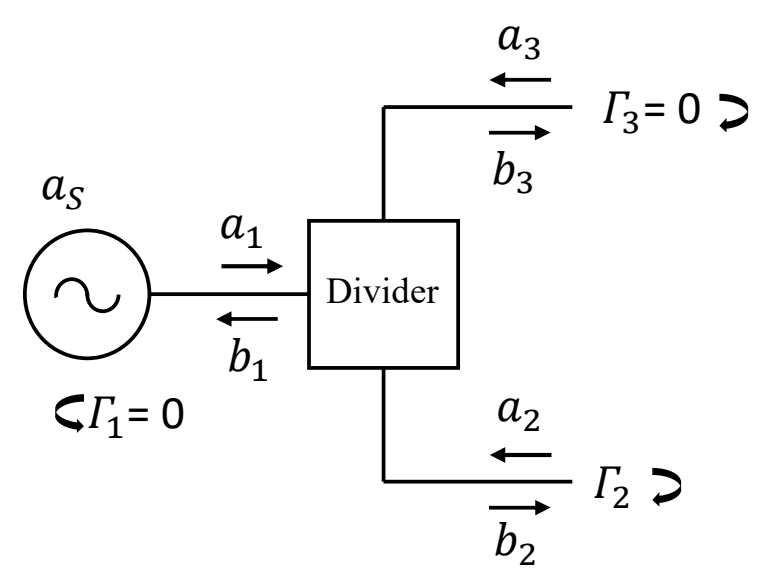

Fig. 5. Diagram a three-port device used in the simplistic derivation. 
As in the previous section, our measured reflection coefficient is

$$
\Gamma^{\prime}=b_{1} / b_{3} .
$$

Next, we assume the termination on port 3 is well-matched such that $a_{3}$ is equal to zero. From (3.7), we can extract the equations for the $b$ waves:

$$
\begin{aligned}
& b_{1}=S_{11} a_{1}+S_{12} a_{2}, \\
& b_{2}=S_{21} a_{1}+S_{22} a_{2},
\end{aligned}
$$

and

$$
b_{3}=S_{31} a_{1}+S_{32} a_{2} .
$$

Solving Eq. (3.10) for $\Gamma_{2}$ gives

$$
\Gamma_{2}=\frac{a_{2}}{b_{2}}=\frac{a_{2}}{S_{21} a_{1}+S_{22} a_{2}}=\frac{1}{S_{21}\left(a_{1} / a_{2}\right)+S_{22}} .
$$

Taking the ratios of Eq. (3.9) and Eq. (3.11) gives $\Gamma^{\prime}$ :

$$
\Gamma^{\prime}=\frac{b_{1}}{b_{3}}=\frac{S_{11} a_{1}+S_{12} a_{2}}{S_{31} a_{1}+S_{32} a_{2}}
$$

or

$$
\Gamma^{\prime}=\frac{S_{11}\left(a_{1} / a_{2}\right)+S_{12}}{S_{31}\left(a_{1} / a_{2}\right)+S_{32}} .
$$

Multiplying both sides of Eq. (3.14) by the denominator of the right-hand side of the equation gives

$$
S_{11}\left(a_{1} / a_{2}\right)+S_{12}=\Gamma^{\prime} S_{31}\left(a_{1} / a_{2}\right)+\Gamma^{\prime} S_{32},
$$

and solving for $a_{1} / a_{2}$ gives

$$
\frac{a_{1}}{a_{2}}=\frac{\Gamma^{\prime} S_{32}-S_{12}}{S_{11}-\Gamma^{\prime} S_{31}} .
$$

Substituting Eq. (3.16) into Eq. (3.12) gives

$$
\Gamma_{2}=\frac{1}{S_{21}\left(\frac{\Gamma^{\prime} S_{32}-S_{12}}{S_{11}-\Gamma^{\prime} S_{31}}\right)+S_{22}}
$$


or

$$
\Gamma_{2}=\frac{\Gamma^{\prime}-S_{11} / S_{31}}{\Gamma^{\prime}\left(S_{22}-\frac{S_{21} S_{32}}{S_{31}}\right)-\frac{1}{S_{31}}\left(S_{11} S_{22}-S_{12} S_{21}\right)} .
$$

Equation (3.18) can be written as

$$
\Gamma_{2}=\frac{\Gamma^{\prime}-e_{00}}{\Gamma^{\prime} \Gamma_{G}-\Delta_{e}}
$$

where,

$$
\begin{gathered}
e_{00}=\frac{S_{11}}{S_{31}} \\
\Delta_{e}=\frac{1}{S_{31}}\left(S_{11} S_{22}-S_{12} S_{21}\right),
\end{gathered}
$$

and

$$
\Gamma_{G}=S_{22}-\frac{S_{21} S_{32}}{S_{31}}
$$

Equation (3.19) is identical to Eq. (3.5), and Eq. (3.22) is the same as Eq. (2.25).

\subsection{Comprehensive Derivation}

In this section, we derive Eq. (3.5) and Eq. (2.25) using a comprehensive model consisting of a three-port device, as shown in Fig. 6, where we explicitly include the reflection coefficients at ports 1 and 3 .

Here, we utilize matrix notation (shown in bold type) to help simplify the math. We begin by expressing the $b$-waves in terms of the scattering matrix of the divider multiplied by the $a$ waves:

$$
\boldsymbol{b}=\boldsymbol{S a} .
$$

The $a$-waves can be expressed as functions of the reflection coefficients multiplied by the $b$ waves plus the contribution of the source:

$$
\boldsymbol{a}=\boldsymbol{\Gamma} \boldsymbol{b}+\boldsymbol{a}_{\boldsymbol{s}}
$$


Substituting Eq. (3.24) into Eq. (3.23) gives

$$
\boldsymbol{b}=\boldsymbol{S}\left(\boldsymbol{\Gamma} \boldsymbol{b}+\boldsymbol{a}_{\boldsymbol{S}}\right),
$$

or

$$
b=S \Gamma b+S a_{S}
$$

Solving for $\boldsymbol{b}$ gives

$$
\boldsymbol{b}=(\boldsymbol{I}-\boldsymbol{S} \boldsymbol{\Gamma})^{-1} \boldsymbol{S} \boldsymbol{a}_{\boldsymbol{S}},
$$

where

$$
\boldsymbol{a}_{s}=\left[\begin{array}{c}
a_{S 1} \\
0 \\
0
\end{array}\right]
$$

The product $\boldsymbol{S} \boldsymbol{a}_{\boldsymbol{s}}$ is

$$
\boldsymbol{S a}_{\boldsymbol{S}}=\left[\begin{array}{lll}
S_{11} & S_{12} & S_{13} \\
S_{21} & S_{22} & S_{23} \\
S_{31} & S_{32} & S_{33}
\end{array}\right]\left[\begin{array}{c}
a_{S 1} \\
0 \\
0
\end{array}\right]=\left[\begin{array}{l}
S_{11} a_{S 1} \\
S_{21} a_{S 1} \\
S_{31} a_{S 1}
\end{array}\right]
$$

and the product $\boldsymbol{S} \boldsymbol{\Gamma}$ is

$$
\boldsymbol{S} \boldsymbol{\Gamma}=\left[\begin{array}{lll}
S_{11} & S_{12} & S_{13} \\
S_{21} & S_{22} & S_{23} \\
S_{31} & S_{32} & S_{33}
\end{array}\right]\left[\begin{array}{ccc}
\Gamma_{1} & 0 & 0 \\
0 & \Gamma_{2} & 0 \\
0 & 0 & \Gamma_{3}
\end{array}\right]=\left[\begin{array}{lll}
\Gamma_{1} S_{11} & \Gamma_{2} S_{12} & \Gamma_{3} S_{13} \\
\Gamma_{1} S_{21} & \Gamma_{2} S_{22} & \Gamma_{3} S_{23} \\
\Gamma_{1} S_{31} & \Gamma_{2} S_{32} & \Gamma_{3} S_{33}
\end{array}\right],
$$

where the off-diagonal elements of the $\boldsymbol{\Gamma}$ matrix are zero since there are no cross-port reflections.

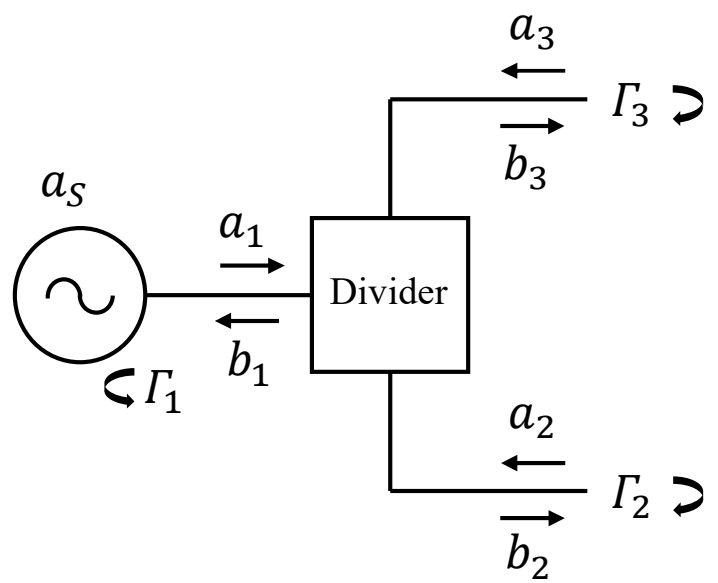

Fig. 6. Diagram a three-port device used in the comprehensive derivation. 
The term $\boldsymbol{I}-\boldsymbol{S} \boldsymbol{\Gamma}$ is

$$
\boldsymbol{I}-\boldsymbol{S} \boldsymbol{\Gamma}=\left[\begin{array}{ccc}
1-\Gamma_{1} S_{11} & -\Gamma_{2} S_{12} & -\Gamma_{3} S_{13} \\
-\Gamma_{1} S_{21} & 1-\Gamma_{2} S_{22} & -\Gamma_{3} S_{23} \\
-\Gamma_{1} S_{31} & -\Gamma_{2} S_{32} & 1-\Gamma_{3} S_{33}
\end{array}\right]
$$

Substituting Eq. (3.29) and Eq. (3.31) into Eq. (3.27) gives

$$
\left[\begin{array}{l}
b_{1} \\
b_{2} \\
b_{3}
\end{array}\right]=\left[\begin{array}{ccc}
1-\Gamma_{1} S_{11} & -\Gamma_{2} S_{12} & -\Gamma_{3} S_{13} \\
-\Gamma_{1} S_{21} & 1-\Gamma_{2} S_{22} & -\Gamma_{3} S_{23} \\
-\Gamma_{1} S_{31} & -\Gamma_{2} S_{32} & 1-\Gamma_{3} S_{33}
\end{array}\right]^{-1}\left[\begin{array}{l}
S_{11} a_{S 1} \\
S_{21} a_{S 1} \\
S_{31} a_{S 1}
\end{array}\right]
$$

where we define $\boldsymbol{A}$ as

$$
\boldsymbol{A}=\left[\begin{array}{ccc}
1-\Gamma_{1} S_{11} & -\Gamma_{2} S_{12} & -\Gamma_{3} S_{13} \\
-\Gamma_{1} S_{21} & 1-\Gamma_{2} S_{22} & -\Gamma_{3} S_{23} \\
-\Gamma_{1} S_{31} & -\Gamma_{2} S_{32} & 1-\Gamma_{3} S_{33}
\end{array}\right]
$$

We can determine the following cofactors of $\boldsymbol{A}$ as

$$
\begin{gathered}
\operatorname{cof}\left(A_{11}\right)=\left(1-\Gamma_{2} S_{22}\right)\left(1-\Gamma_{3} S_{33}\right)-\Gamma_{2} \Gamma_{3} S_{23} S_{32} \\
\operatorname{cof}\left(A_{21}\right)=\Gamma_{2} S_{12}\left(1-\Gamma_{3} S_{33}\right)+\Gamma_{2} \Gamma_{3} S_{13} S_{32} \\
\operatorname{cof}\left(A_{31}\right)=\Gamma_{2} \Gamma_{3} S_{12} S_{23}+\Gamma_{3} S_{13}\left(1-\Gamma_{2} S_{22}\right) \\
\operatorname{cof}\left(A_{13}\right)=\Gamma_{1} \Gamma_{2} S_{21} S_{32}+\Gamma_{1} S_{31}\left(1-\Gamma_{2} S_{22}\right) \\
\operatorname{cof}\left(A_{23}\right)=\Gamma_{2} S_{32}\left(1-\Gamma_{1} S_{11}\right)+\Gamma_{1} \Gamma_{2} S_{12} S_{31}
\end{gathered}
$$

and

$$
\operatorname{cof}\left(A_{33}\right)=\left(1-\Gamma_{1} S_{11}\right)\left(1-\Gamma_{2} S_{22}\right)-\Gamma_{1} \Gamma_{2} S_{12} S_{21}
$$

The terms $b_{1}$ and $b_{3}$ in Eq. (3.32) can then be extracted as

$$
b_{1}=\frac{1}{\operatorname{det}(A)}\left[S_{11} \operatorname{cof}\left(A_{11}\right)+S_{21} \operatorname{cof}\left(A_{21}\right)+S_{31} \operatorname{cof}\left(A_{31}\right)\right] a_{S 1}
$$

and

$$
b_{3}=\frac{1}{\operatorname{det}(A)}\left[S_{11} \operatorname{cof}\left(A_{13}\right)+S_{21} \operatorname{cof}\left(A_{23}\right)+S_{31} \operatorname{cof}\left(A_{33}\right)\right] a_{S 1} \text {. }
$$

The measured reflection coefficient $\Gamma^{\prime}$ is the ratio of $b_{1}$ and $b_{3}$ : 


$$
\Gamma^{\prime}=\frac{b_{1}}{b_{3}}=\frac{S_{11} \operatorname{cof}\left(A_{11}\right)+S_{21} \operatorname{cof}\left(A_{21}\right)+S_{31} \operatorname{cof}\left(A_{31}\right)}{S_{11} \operatorname{cof}\left(A_{13}\right)+S_{21} \operatorname{cof}\left(A_{23}\right)+S_{31} \operatorname{cof}\left(A_{33}\right)} .
$$

Substituting Eqs. (3.34-3.39) into Eq. (3.42) gives

$$
\Gamma^{\prime}=\frac{\left\{\begin{array}{c}
S_{11}\left[\left(1-\Gamma_{2} S_{22}\right)\left(1-\Gamma_{3} S_{33}\right)-\Gamma_{2} \Gamma_{3} S_{23} S_{32}\right] \\
+S_{21}\left[\Gamma_{2} S_{12}\left(1-\Gamma_{3} S_{33}\right)+\Gamma_{2} \Gamma_{3} S_{13} S_{32}\right] \\
+S_{31}\left[\Gamma_{2} \Gamma_{3} S_{12} S_{23}+\Gamma_{3} S_{13}\left(1-\Gamma_{2} S_{22}\right)\right]
\end{array}\right\}}{\left\{\begin{array}{c}
S_{11}\left[\Gamma_{1} \Gamma_{2} S_{21} S_{32}+\Gamma_{1} S_{31}\left(1-\Gamma_{2} S_{22}\right)\right] \\
+S_{21}\left[\Gamma_{2} S_{32}\left(1-\Gamma_{1} S_{11}\right)+\Gamma_{1} \Gamma_{2} S_{12} S_{31}\right] \\
+S_{31}\left[\left(1-\Gamma_{1} S_{11}\right)\left(1-\Gamma_{2} S_{22}\right)-\Gamma_{1} \Gamma_{2} S_{12} S_{21}\right]
\end{array}\right\}} .
$$

Equation (3.43) can be expressed as

$$
\Gamma^{\prime}=\frac{\alpha \Gamma_{2}+\beta}{\gamma \Gamma_{2}+\theta},
$$

where

$$
\begin{gathered}
\alpha=\left(S_{12} S_{21}-S_{11} S_{22}\right)+\Gamma_{3}\left(\begin{array}{c}
S_{11} S_{22} S_{33}-S_{11} S_{23} S_{32}-S_{12} S_{21} S_{33} \\
+S_{12} S_{23} S_{31}+S_{13} S_{21} S_{32}-S_{13} S_{22} S_{31}
\end{array}\right), \\
\beta=S_{11}+\Gamma_{3}\left(S_{13} S_{31}-S_{11} S_{33}\right), \\
\gamma=S_{21} S_{32}-S_{22} S_{31}
\end{gathered}
$$

and

$$
\theta=S_{31}
$$

Equation (3.44) can be rearranged as

$$
\Gamma_{2}=\frac{\Gamma^{\prime}-\left(\frac{\beta}{\theta}\right)}{\Gamma^{\prime}\left(-\frac{\gamma}{\theta}\right)-\left(\frac{\alpha}{\theta}\right)} .
$$

Comparing Eq. (3.49) with Eq. (3.19), we see that the equivalent source mismatch term $\Gamma_{G}$ is

$$
\Gamma_{G}=-\frac{\gamma}{\theta} .
$$

Substituting Eq. (3.47) and Eq. (3.48) into Eq. (3.50) gives

$$
\Gamma_{G}=-\frac{S_{21} S_{32}-S_{22} S_{31}}{S_{31}},
$$


or

$$
\Gamma_{G}=S_{22}-\frac{S_{21} S_{32}}{S_{31}}
$$

Equation (3.52) is identical to Eq. (3.22). From this comprehensive derivation, we can see that $\Gamma_{G}$ is independent of $\Gamma_{1}$ and $\Gamma_{3}$. This result is consistent with Juroshek's conclusion [5].

\section{Conclusions}

We have provided derivations for the equations that govern the direct-comparison system utilizing scattering-parameter flow diagrams and power definitions. Additionally, we specified a correction term for the case when an adapter is connected to the known power sensor. Finally, we reviewed the most widely-accepted approach for determining the equivalent source reflection coefficient of a three-port device, such as a power divider, and provided derivations that demonstrate its independence of external impedance mismatches at two of its ports.

\section{Acknowledgments}

The authors thank Jim Booth, Ari Feldman, Paul Hale, Mitch Wallis, and Dylan Williams for their helpful comments.

\section{References}

[1] Clague FR, Voris PG (1993) Coaxial reference standard for microwave power (National Institute of Standards and Technology, Boulder, CO), NIST Technical Note (TN)1357.

[2] Weidman MP (1996) Direct comparison transfer of microwave power sensor calibrations (National Institute of Standards and Technology, Boulder, CO), NIST Technical Note (TN) 1379.

[3] Juroshek JR (2000) NIST 0.05-50 GHz direct-comparison power calibration system. Conference on Precision Electromagnetic Measurements, pp. 166-167.

[4] Rytting D (1987) Advances in microwave error correction techniques. $R F$ and Microwave Measurement Symposium.

[5] Juroshek JR (1997) A direct calibration method for measuring equivalent source mismatch. Microwave Journal, pp. 106-118.

[6] Kerns DM, Beatty RW (1967) Basic Theory of Waveguide Junctions and Introductory Microwave Network Analysis (Pergamon Press).

[7] Keysight Technologies (2104) Fundamentals of RF and Microwave Power Measurements (Part 3). Application Note 1449-3, 5988-9215EN.

[8] Teppati V, Ferrero A, Sayed M (2013) Modern RF and Microwave Measurement Techniques (Cambridge University Press).

[9] Engen GF (1958) Amplitude stabilization of a microwave signal source. IRE Transactions on Microwave Theory and Techniques, 6(2): 202-206.

[10] Fantom A (1990) Radio Frequency and Microwave Power Measurement (Peter Peregrinus). 
[11] Wallis TM, Crowley TP, LeGolvan DX, Ginley RA (2012) A direct comparison system for power calibration up to $67 \mathrm{GHz}$. Conference on Precision Electromagnetic Measurements, pp. 726-727.

[12] Kang TW, Kim JH, Kwon JY, Park JI, Lee DJ (2012) Direct comparison technique using a transfer power standard with an adapter and its uncertainty. Conference on Precision Electromagnetic Measurements, pp. 728-729. 